\title{
Instrumento para classificação do grau de dependência de usuários: um estudo para contribuir no dimensionamento de pessoal
}

Recebido em: 13/06/2011

Aceito em: 14/02/2012

\author{
Francine Lima Gelbcke ${ }^{1}$ \\ Eliane Matos ${ }^{2}$ \\ Roseli Schmoeller ${ }^{3}$ \\ Maria Patrícia Locks de Mesquita ${ }^{4}$ \\ Silvana Alves Benedet ${ }^{5}$
}

Pesquisa que visa a testar instrumento de classificação de usuários, adaptada do modelo de Fugulin et al. (2005) e ampliada com a inclusão dos itens: regulação hormonal; educação em saúde/comunicação; sono/repouso; segurança emocional; percepção dos órgãos dos sentidos; e família/acompanhante ou gregária. Foi aplicada em unidades de internação pelo período de dois meses e apontou: necessidade de aprimorar os subitens avaliados, explicitando cada necessidade, e distribuição de cinco graus de avaliação: mínimo, intermediário, alta dependência, semi-intensivo e intensivo. A proposta mostrou-se adequada à avaliação do fazer da enfermagem, contribuindo no estudo da carga de trabalho para o dimensionamento do pessoal de enfermagem.

Descritores: Serviços de Saúde, Dimensionamento de Pessoal, Administração de Serviços de Saúde.

Instrument for grading the degree of dependence of users: a study to contribute to the dimensionig of personal

This research aims to test users' classification instrument, adapted from the model Fugulin et al (2005), and expanded with the inclusion of the items: hormonal regulation, health education/communication, sleep/rest, emotional security, perception of senses organs, and family/caregivers or gregarious. It was applied at internment units for a period of two months and pointed out: the need to improve the sub items, explained each assessed need, distribution of five degrees of evaluation: minimum, intermediate, high dependency, intensive and semi-intensive. The proposal proved to be suitable for assessment of the nursing activity, contributing to the evaluation of the workload for the dimensioning of nursing staff.

Descriptors: Health Services, Personnel Dimensioning, Health Services, Administration.

Instrumiento para clasificación del grado de dependência de usuários: un estudio para contribuir en el dimensionamiento de personal Se trata de una investigación que tiene por objectivo testar instrumento de clasificación de usuarios, adaptado del modelo de Fugulin et al (2005), y ampliado con inclusión de los aspectos: regulación hormonal, educación en salud/comunicación; sueno/repuso; aseguranza emocional; percepción de los órganos de los sentidos; y familia/acompañante o gregario. Se aplicó a las unidades de hospitalización por un período de dos meses e apunto: necesidad de mejorar los subitens evaluados explicitando cada necesidad, distribución de cinco grados de evaluación: mínimo, intermediario, alta dependencia, semiintensivo e intensivo. La propuesta consistía en una evaluación adecuada de la enfermería, lo que contribuye a la evaluación de la carga de trabajo para el cálculo del personal de enfermería.

Descriptores: Servicios de Salud, Dimensionamiento de Personal, Administración los Servicios de Salud.

\section{INTRODUÇÃO}

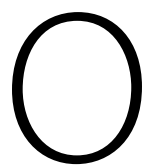
dimensionamento da força de trabalho em saúde é uma área importante na definição das políticas de gestão de pessoas para o Sistema Único de Saúde (SUS), pois os recursos humanos constituem a base dos serviços de saúde e do próprio sistema. $\mathrm{Na}$ área da saúde e, em especial, na enfermagem, o dimensionamento tem se constituído um foco de atenção dos gestores de serviço de saúde, por interferir na eficácia, na qualidade e no custo da atenção à saúde.

A gestão de recursos humanos envolve uma complexidade significativa de fatores, pois a força de trabalho é protagonista nos processos de produção, não podendo mais ser entendida como um simples recurso. Apesar de sua relevância nos processos produtivos, ainda se encontra uma inadequação na distribuição dos profissionais, em diferentes níveis de atenção à saúde, por categoria profissional ou por região, implicando no aumento de custos, limitando o acesso da população aos serviços de saúde, bem como interferindo na qualidade da assistência prestada ${ }^{(1-4)}$.

Há que se considerar que "a gestão do trabalho nos

1 Enfermeira, Diretora de Enfermagem do Hospital Universitário da Universidade Santa Catarina (HU/UFSC). Doutora em Enfermagem. Professora do Departamento. de Enfermagem e do Programa de Pós-Graduação de Enfermagem (PEN/UFSC). Vice-líder do Grupo Práxis/PEN/UFSC.

2 Enfermeira do HU/UFSC. Doutora em Enfermagem. Membro do Grupo PRAXIS. Professora credenciada pelo Mestrado Profissional em Enfermagem - PEN-UFSC.

3 Enfermeira do HU/UFSC. Mestranda do PEN/UFSC. Membro do Grupo PRAXIS.

4 Enfermeira do HU/UFSC. Mestre em Enfermagem. Doutoranda do PEN/UFSC. Membro do Grupo Práxis.

5 Enfermeira do HU/UFSC. Mestre em Enfermagem. 
serviços de saúde adquire conotações peculiares em função do significado especial que tem o trabalho no processo de produção desses serviços. Lidar com o trabalho em saúde tem implicações especiais porque não se trata apenas de uma mercadoria cujo valor se estipule de acordo com as regras de mercado, ou mesmo de um fator de produção cuja utilização se estabeleça conforme regulamentos cristalizados em leis"(5:10).

A gestão de recursos humanos torna-se um componente importante na definição das políticas públicas, exigindo, para seu dimensionamento, mais do que a aplicação de fórmulas matemáticas, a avaliação das peculiaridades de cada setor, considerando os diversos problemas de saúde que acometem a população, que têm caráter múltiplo e envolvem as particularidades dos usuários do sistema, os quais são indivíduos únicos e multidimensionais. Essas particularidades interferem no processo de trabalho, indicando variedade, complexidade e diversidade de situações, com especificidades regionais e mesmos locais, com graus de cuidado diversificados, desde a atenção básica até a de alta complexidade, exigindo uma força de trabalho diferenciada em número e qualificação, o que dificulta uma padronização ${ }^{(1,6)}$. Portanto, somente $\mathrm{o}$ avanço tecnológico não responde às necessidades individuais e coletivas, e os usuários dos serviços de saúde, hoje mais atentos a seus direitos, exigem uma força de trabalho qualificada e que dê conta de sua atenção, implicando em uma rede complexa de relações que envolvem os diversos profissionais que atuam nos serviços de saúde e os usuários do sistema $^{(2-4)}$.

No que diz respeito ao dimensionamento de pessoal de enfermagem, os cálculos matemáticos atualmente utilizados nos diversos estudos estão pautados em aspectos importantes, como jornada e carga de trabalho e índice de absenteísmo, porém outras condições devem ser consideradas, como característica da instituição, perfil epidemiológico da demanda, aparato tecnológico, educação permanente, atividades que não são consideradas como de assistência direta ao usuário, e, portanto, não incluídas nos cálculos ${ }^{(1,6-8)}$.

O dimensionamento de pessoal é um assunto complexo, situado em uma área de conflito de interesses entre custobenefício, pessoal-institucional, capital-trabalho e técnicoético. A abordagem do dimensionamento de pessoal, mesmo na esfera técnica, é um tema polêmico, porém necessário, face às mudanças constantes e rápidas que vêm ocorrendo no setor de saúde, implicando em adequações conscientes da força de trabalho. A atualização e discussão permanente do dimensionamento de pessoal coloca-se como um instrumento gerencial significativo ${ }^{(7,8)}$.

$\mathrm{Na}$ prática, o planejamento quantitativo de recursos humanos $(\mathrm{RH})$ de enfermagem tem sido realizado de forma empírica, baseado na experiência e no julgamento dos enfermeiros, que têm encontrado inúmeras dificuldades para planejar e justificar a necessidade de adequação no quantitativo de recursos humanos. Isso ocorre tanto para a melhoria da qualidade assistencial quanto para o atendimento das novas demandas impostas pelos administradores das instituições de saúde, frente à necessidade crescente de racionalizar os custos e aumentar a oferta de serviços ${ }^{(9,10)}$. A caracterização dos níveis de cuidado é um aspecto importante para a determinação da carga de trabalho da unidade, sendo que, para as unidades de internação hospitalar, essa variável pode ser identificada por meio de adoção de um Sistema de Classificação de Pacientes $(\mathrm{SCP})^{(8,9)}$. Há que se salientar que o SCP utilizado em vários estudos comporta, na atualidade, os aspectos biológicos, como estado mental, oxigenação, sinais vitais, motilidade, deambulação, alimentação, cuidado corporal, eliminação e terapêutica ${ }^{(8,9,11)}$. É necessário avançar em propostas que incluam também os aspectos psicossociais.

Como parte de um estudo que tem como objetivo geral propor uma metodologia para Dimensionamento Quanti-Qualitativo de Pessoal de Enfermagem (DQQPE) em unidades de internação de Hospitais Universitários Federais, este trabalho visa a caracterizar os níveis de cuidado necessários aos usuários em unidades de internação, considerando as diversas dimensões dos sujeitos do cuidado.

\section{METODOLOGIA}

Entende-se como carga de trabalho da unidade de enfermagem o produto da quantidade média diária de usuários assistidos, segundo o número de trabalhadores, pelo tempo médio de assistência de enfermagem utilizada, por usuário, de acordo com o grau de dependência e atendimento realizado ${ }^{(9)}$.

Avaliando-se os aspectos constantes no $\mathrm{SCP}(8,9)$ utilizado na atualidade pela enfermagem brasileira, elaborou-se um instrumento, adaptado a partir desse, incluindo a avaliação dos fatores: integridade cutâneo mucosa, educação/ comunicação, sono e repouso, segurança emocional e percepção dos órgãos dos sentidos, na determinação da quantidade/qualidade da assistência de enfermagem necessária para os usuários de um hospital-escola.

Inicialmente, foram incluídos os aspectos acima à classificação de pacientes existentes, o que resultou em um instrumento no qual constam 17 itens de avaliação.

Para classificar o usuário de acordo com o nível de cuidado - mínimo, intermediário, semi-intensivo e intensivo -, o instrumento foi quantificado para dar visibilidade ao nível de cuidado em cada unidade de internação. $O$ escore do instrumento utilizado para a classificação do grau de 
dependência do usuário considerou os seguintes valores: cuidado mínimo - a soma de metade do valor do grau l e grau II $(8+16)$, estabelecendo-se para grau mínimo um escore até 24 pontos; cuidado intermediário - a soma de metade do valor do grau II e grau III $(16+24)$, estabelecendo-se para grau intermediário um escore de 25 até 40 pontos; cuidado semi-intensivo - a soma de metade do valor do grau III e grau IV ( $24+32)$, estabelecendo-se para grau semi-intensivo um escore de 41 até 56 pontos; cuidados intensivos - acima de 57 pontos.

Em um primeiro momento, o instrumento foi aplicado por estudantes de graduação em enfermagem do sétimo período, acompanhados por um dos pesquisadores, como etapa de testagem do mesmo, a todos os usuários internados no momento dos estágios dos alunos, durante um período de 30 dias, na unidade de pediatria, de neonatologia e no repouso da emergência de adulto.

Posteriormente, o instrumento foi aplicado pelos enfermeiros das unidades de internação da instituição, após orientação, por um período mínimo de dois meses, a todos os usuários internados, em duas unidades piloto (clínica médica e clínica cirúrgica).

A adequação do instrumento proposto foi analisada considerando os resultados da aplicação na fase de teste e a aplicação posterior pelos enfermeiros, as dificuldades e facilidades relatadas pelos enfermeiros e pesquisadores, as sugestões de mudanças no instrumento, a viabilidade de utilização na prática.

A pesquisa obedeceu aos aspectos éticos nos termos das resoluções 196/96 e 251/97, do Conselho Nacional de Saúde sendo aprovada pelo Comitê de Ética em Pesquisa em Seres Humanos da UFSC, mediante o parecer 273/07.

\section{O OLHAR PARA A AVALIAÇÃO DO GRAU DE DEPENDÊNCIA DOS PACIENTES - PRIMEIROS RESULTADOS}

O dimensionamento do pessoal de enfermagem deve produzir resultados que possibilitem alcançar um quadro adequado às necessidades da clientela, sendo definido como "etapa inicial do processo de provimento de pessoal, que tem por finalidade a previsão de quantidade de funcionários por categoria, requerido para suprir as necessidades de assistência de enfermagem, direta ou indiretamente prestada à clientela"(10).

Os estudos sobre dimensionamento de pessoal de enfermagem indicam a necessidade de dados, que devem ser coletados durante um período que varia entre três meses e um ano, sendo que, para avaliar o número de horas de assistência de enfermagem, os instrumentos preveem uma série de dados relativos à dimensão biológica dos usuários dos serviços de saúde. Não são considerados nessa análise aspectos subjetivos que interferem no cuidado desse sujeito em sua integralidade e multidimensionalidade e influenciam o quantitativo de pessoal de enfermagem para prestação da assistência( ${ }^{(8,9)}$

Nesse sentido, a avaliação do grau de dependência incluindo os aspectos relacionados às necessidades humanas básicas de integridade cutâneo-mucosa; a educação em saúde/comunicação; o sono e repouso; a regulação hormonal, principalmente no que se refere à avaliação de hiper e hipoglicemia; a segurança emocional; a percepção dos órgãos dos sentidos, considerando-se audição, visão, tato e dor; e a condição da família/acompanhante ou gregária no cuidado contribuiu para uma avaliação mais abrangente dos usuários, permitindo uma visão mais ampliada do usuário e de suas necessidades assistenciais. Possibilitou também um olhar diferenciado para o fazer da enfermagem.

Vários são os benefícios da utilização do sistema de classificação de usuários, quer na qualidade do cuidado prestado devido à individualização das necessidades de cada sujeito, no planejamento da assistência, quer na construção de um banco de dados que auxiliam na tomada de decisões, no monitoramento da produtividade e inclusive nos custos dos serviços de enfermagem ${ }^{(12)}$. Porém, para que efetivamente a utilização da classificação alcance tais benefícios, entendemos que o usuário necessite ser avaliado integralmente, nos aspectos biopsicossociais.

$\mathrm{Na}$ avaliação dos resultados obtidos pela classificação dos usuários com base no instrumento proposto, os enfermeiros verificaram que esse contemplou aspectos da dimensão subjetiva do cuidado e proporcionou uma avaliação mais fidedigna do grau de dependência e a necessidade real de cuidado de enfermagem, segundo os padrões de assistência da instituição de ensino estudada.

$O$ instrumento desenvolvido permitiu um melhor detalhamento das necessidades do usuário em relação a cada grau de dependência, embora tenham sido apontadas necessidades de seu aprimoramento, que surgiram principalmente dos enfermeiros. Os acadêmicos de enfermagem, em função da pouca vivência com a profissão, não sinalizaram para reformulações significativas.

A proposta de instrumento para a classificação de usuários foi considerada extensa e detalhada, porém de fácil assimilação pelos avaliadores. Nesse sentido, embora tenha implicado em um gasto maior de tempo dos enfermeiros para sua aplicação, esse tempo foi revertido em benefício da equipe de trabalho e do próprio usuário, por avaliar outras questões que perpassam o cuidado.

Entre os aspectos a serem reformulados, os enfermeiros destacaram alterações nos subitens avaliados em cada necessidade, dando maior clareza ao processo. Foram apontados, também, déficits no que se refere à pontuação que define o grau de cuidado, corroborando com estudos 
que propõem a ampliação de quatro para cinco os graus de dependência adotados pelo Cofen, ou seja, introduzindo o nível de alta dependência de complexidade de cuidado $(9,13)$. Considerando esses aspectos, o instrumento está passando por uma avaliação minuciosa, que inclui a proposta de ampliação dos níveis de cuidado e a adequada inserção dos aspectos psicossociais na avaliação dos usuários.

Outro aspecto importante que está em fase de construção é a interface do instrumento de classificação do usuário com a etapa de evolução informatizada de enfermagem, constante na sistematização da assistência, de modo a permitir a associação entre a evolução de enfermagem e a classificação de cada paciente assistido nas unidades de internação, diariamente.

O aprimoramento do sistema de classificação dos usuários, com a inclusão dos aspectos propostos e a informatização desse instrumento associado à sistematização da assistência de enfermagem, apresenta, segundo avaliação inicial, potencial para aperfeiçoar o dimensionamento de pessoal de enfermagem e para uma assistência mais qualificada e segura, impactando o resultado assistencial ${ }^{(14)}$.

\section{CONCLUSÕES}

A experiência de elaboração e aplicação de um instrumento de classificação de usuários voltado para a realidade de um hospital-escola traz importantes contribuições para a produção do conhecimento na área da gestão de pessoas, principalmente nesta que foi considerada a década de recursos humanos. Do mesmo modo, a proposta de ampliação desse instrumento, incluindo aspectos relacionados às necessidades psicossociais, pode futuramente contribuir para uma melhor classificação do grau de atenção aos usuários, ao associar aspectos que ultrapassam os aspectos biológicos, dando a devida dimensão do cuidar.

Como proposta preliminar, entendese que a aplicação do instrumento possibilitou uma avaliação mais efetiva das perspectivas que envolvem - cuidado de enfermagem aos usuários de um hospital-escola. O escore encontrado na aplicação do instrumento modificou em algumas situações o nível de complexidade do cuidado, mostrando necessidades dos usuários que demandam atenção de enfermagem e dando visibilidade a aspectos do trabalho que não são percebidos quando esse está voltado exclusivamente para a dimensão biológica. $O$ instrumento desenvolvido mostrou aplicabilidade em unidades de internação cirúrgica, médica, pediátrica e de neonatologia.

Expandir a avaliação dos usuários, com uma melhor classificação do grau de atenção, permite aumentar a dimensão do cuidar em sua integralidade. Entende-se que a aplicação desse instrumento ampliado poderá contribuir para o atendimento da demanda das instituições de saúde e ensino, bem como embasar o planejamento e justificar a necessidade de adequação no quantitativo de recursos humanos, para o dimensionamento de pessoal de enfermagem mais efetivo e real.

\section{Referências}

1. Nicola AL. Dimensionamento de pessoal de enfermagem no Hospital

Universitário do Oeste do Paraná [tese]. Ribeirão Preto: Escola de Enfermagem da USP; 2004.

2. Mendes IAC, Marziale MHP. Década de recursos humanos em saúde: 2006-2015. Rev Latinoam Enferm. 2006;14(1):1-2.

3. Organização Mundial de Saúde. Colaboremos por la salud. Informe sobre la salud en el mundo 2006. Genebra: OMS; 2006.

4. Ministério da Saúde (BR). Política de recursos humanos em saúde. Brasilia:

Ministério da Saúde; 2002.

5. Campos FE, Santana JP. Introdução. In: Ministério da Saúde (BR). Política de recursos humanos em saúde. Brasília: Ministério da Saúde; 2002. p. 9-11. 6. Santana JP. A gestão do trabalho nos estabelecimentos de saúde: elementos para uma proposta. II Conferência Nacional de Recursos Humanos de Saúde. Brasilia: [s.n.]; 1993.

7. Campos LF. Dimensionamento de pessoal de enfermagem nos hospitais de Ribeirão Preto-SP [dissertação]. Ribeirão Preto: Escola de Enfermagem da USP; 2004

8. Gaiszinski RR. O dimensionamento do pessoal de enfermagem em instituiçöes hospitalares [tese de livre-docência]. São Paulo: Escola de Enfermagem da USP; 1998. 9. Fugulin FMT, Gaidzinki RR, Kurcgant P. Sistema de classificação de pacientes: identificaçăo do perfil assistencial dos pacientes das unidades de internação do HU-USP. Rev Latinoam Enferm. 2005;13(1):72-8.

10. Kurcgant P. Gerenciamento em enfermagem. Rio de Janeiro: Guanabara Koogan; 2005.

11. Matsushita MS, Adami NP, Carmagnani MIS. Dimensionamento do pessoal de enfermagem das unidades de internação do Hospital São Paulo. Acta Paul Enferm. 2005;18(1):9-19.

12. Pagliarini FC, Perroca MG. Uso de instrumento de classificação de pacientes como norteador do planejamento de alta de enfermagem. Acta Paul Enferm. 2008;21(3):393-7

13. Santos F, Rogenski NMB, Baptista CMC, Fugulin FMT. Sistema de classificação de pacientes: proposta de complementação do instrumento de Fugulin et al. Rev Latinoam Enferm. 2007;15(5).

14. Gaidzinski RR, Fugulin FMT. Condições de trabalho e segurança do profissional: a influência do dimensionamento dos trabalhadores de enfermagem. In: Anais do $62^{\circ}$ Congresso Brasileiro de Enfermagem. Florianópolis, 2010. 